\title{
QUELQUES DIPTÈRES VULNÉRANTS DU GABON
}

\author{
Par H. GALLIARD
}

Au cours d'un séjour dans le Gabon occidental, dans la région située dans le quadrilatère formé par l'Ogooué au nord, la Nyanga au sud, l'Ogooué à l'est et la mer à l'ouest, j'ai pu récolter un certain nombre de diptères vulnérants. Je n'envisagerai ici que les tabanidés, les simulidés et les cératopogoninés, les autres diptères (culicidés, phlébotomes, glossines) ayant fait ou devant faire l'objet de notes séparées.

Tabanidés. - Dans la région où j'ai prospecté, j'ai pu recueillir les spécimens suivants (1):

Tabanus par Walker.

T. thoracinus P. de Beauvois.

T. secedens Walker.

T. besti Surc., var. arbucklei Austen.

T. argenteus Surcouf.

T. roubaudi Surcouf.

T.fasciatus, var. nigripes Surcouf.

T. congolensis Ricardo.
T. obscurefumatus Surcouf.

T. copemanni Austen.

T. atrimanus Lœw.

T. pertinens Austen.

Hippocentrum strigipenne Karsch. Hæmatopota harpax Austen.

H. insatiabilis Austen.

Chrysops dimidiata v. der Wulp.

Les Tabanidés sont très abondants en certains points de la forêt et sur les fleuves jusqu'au 15 juin, en saison sèche. Hæmatopota harpax était particulièrement fréquent sur les bords de la Nyanga et dans quelques localités forestières. C'est la seule espèce que j'ai rencontrée en forêt, pendant la saison sèche.

Je n'ai rencontré qu'une espèce de Chrysops, $C$. dimidiata, appelée diwouego oudibenga en langue bapounou. Les premiers spécimens ont été capturés vers le 5 septembre, avant les premières fortes pluies, entre Eschiras et Bongo, dans le Mayumbe. Son vol est lent et lourd, il est facile à capturer. A partir de ce moment le nombre augmente progressivement et on le trouve un peu partout, surtout en savane et le long des fleuves.

Simulies. - Les espèces récoltées appartiennent à l'espèce Simulium damnosum. J'ai signalé (2) que dans les régions que j'avais par-

(1) Ces tabanides ont été aimablement déterminées par Miss A. M. Evans et les culicoïdes par le prof. Patton, de l'Ecole de Médecine tropicale de Liverpool.

(2) Recherches sur la filariose au Gabon occidental. Bull. Soc. Path. exot., 1931 , p. 167.

Annales de Parasitologie, T. XI, $\mathrm{N}^{\circ} 1 .-1^{\mathrm{er}}$ janvier 1933 , p. 24-25. 
courues l'onchocercose n'était pas très fréquente. Les simulies ne se rencontrent que par place, au début de la saison des pluies, mais toujours en très petit nombre, rarement en forêt, mais plus souvent le long des cours d'eau de savane. Du mois de juin au 15 septembre, il m'a été très difficile d'en découvrir.

Gulicoides. - Les spécimens récoltés appartiennent à l'espèce Culicoides grahami. Ces insectes ne sont pas très répandus. En saison sèche, on ne les rencontre que dans les localités, toujours les mêmes, bien connues des blancs, qui les redoutent particulièrement. Ils sont désignés ici sous le nom de fourous, mais ce nom est importé, les indigènes ne le connaissent pas ; il sert à désigner aux colonies tantôt les Simulium, tantôt les Culicoides. Ici les premiers étant rares, ce sont les seconds qui héritent du nom (1).

C'est surtout vers le soir, un peu avant le coucher du soleil, que les fourous apparaissent en véritables essaims. Mais ils disparaissent dès que la nuit tombe. Ils ne piquent jamais au cours de la nuit, contrairement à ce qu'a constaté Schwetz. Il est curieux d'observer, à propos de ces insectes, combien les individus réagissent différemment aux piqûres. Au Congo belge, la piqûre est douloureuse; ici elle passe absolument inaperçue, mais en quelques secondes, le visage, le cou, les bras, sont recouverts de tâches rosées, arrondies, devenant légèrement papuleuses, ecchymotiques le lendemain, et persistant 24 à 48 heures. Chez les uns, la lésion devient prurigineuse au bout de quelques minutes, chez d'autres elle ne le devient que 6 ou 8 heures après.

Les localités sont: Forêt du Mayumbe entre Mayumba et Tchibanga (village de Goumbo), juin ; forêt entre Eschiras et Bongo (plusieurs localités, surtout village M'bengui), septembre.

Auchmeromyia luteola. - La larve de cette mouche est désignée sous les noms de baguingui baboutamba en langue bapounou et m'fingui tsiboutoho en langue baloumbo. Cette larve hématophage se rencontre fréquemment, surtout dans la N'gounié. Elle était remarquablement abondante dans les cases des trypanosomés du poste de Mouila.

Parmi les arthropodes piqueurs, j'ai recherché particulièrement les Ornithodorus. A la vue des spécimens que j'avais emporté, de nombreux indigènes disaient les connaître, sous le nom de sikoufa. Je n'ai jamais pu cependant en obtenir un seul exemplaire.

Laboratoire de Parasitologie de la Faculté de médecine de Paris.

(1) D'après Schwetz au Congo belge, le mot « maringouin » sert comme le mot "fourou ", à désigner toutes les petites bêtes qui piquent. Bull. Soc. Path. exot., XXIII, 1930, p. 987. 